\title{
Relativistic quantum mechanical calculations of electron-impact broadening for spectral lines in Be-like ions
}

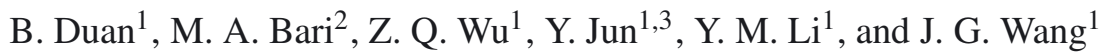 \\ ${ }^{1}$ Data Center for High Energy Density Physics, Institute of Applied Physics and Computation Mathematics, 100088 Beijing, \\ PR China \\ e-mail: alexduan1967@hotmail.com, duan_bin@iapcm.ac.cn \\ 2 Pakistan Atomic Energy Commission, PO Box 1114, 44000 Islamabad, Pakistan \\ ${ }^{3}$ HEDPS, Center for Applied Physics and Technology, Peking University, 100871 Beijing, PR China
}

Received 8 November 2011 / Accepted 6 August 2012

\begin{abstract}
Aims. We present relativistic quantum mechanical calculations of electron-impact broadening of the singlet and triplet transition $2 \mathrm{~s} 3 \mathrm{~s} \leftarrow 2 \mathrm{~s} 3 \mathrm{p}$ in four Be-like ions from N IV to Ne VII.

Methods. In our theoretical calculations, the K-matrix and related symmetry information determined by the colliding systems are generated by the DARC codes.

Results. A careful comparison between our calculations and experimental results shows good agreement. Our calculated widths of spectral lines also agree with earlier theoretical results. Our investigations provide new methods of calculating electron-impact broadening parameters for plasma diagnostics.
\end{abstract}

Key words. line: profiles - atomic processes - atomic data

\section{Introduction}

Generally, radiation damping of an excited atom leads to broadening of spectral lines. In dense plasmas, radiation damping stems from the interactions of an emitting atom or ion with perturbing plasma constituents. Among these interactions, the electron colliding with ions can lead to excitations and broadening of ionic spectral lines. The electron-impact broadening is a major contributor to radiative acceleration in stellar interiors and is of particular importance as a diagnostic tool in high-temperature plasmas. Not only is it important to precision spectroscopy, but also reveals dependence on major plasma characteristics, such as density and temperature. In the case of plasma diagnostics, the careful analysis of line profiles is a powerful technique for studying atomic and molecular interactions. The knowledge of electron-impact broadening is paramount for probing matter under extreme physical conditions such as in stellar atmospheres. In stellar interiors, electron-impact broadening contributes significantly to radiative acceleration, because of high electron density, and is imperative for evolutionary models, atomic abundances, and radiative opacity calculations.

To study electron-impact broadening, several theoretical methods and techniques have been used in the literature (Griem 1974; Dimitrijević \& Konjević 1980). However, there have been very few quantum mechanical calculations of electron-impact broadening and shift in spectral lines. Recently, the full quantum mechanical calculations for electron impact broadening of the $2 s 3 s-2 s 3 p$ transitions for the four Be-like ions from N IV to Ne VII have been performed by Elabidi et al. (2008). These calculations are based on the nonrelativistic quantum mechanics and intermediate coupling. In the context of quantum mechanics, electron-impact broadening and shift of spectral lines is studied by two different methods, i.e., calculating the scattering cross sections and generating the $\mathbf{K}$-matrix. In the present work, we adopt the latter approach, defined by calculating $\mathbf{K}$-matrix, which is similar to the method adopted by Seaton (1987).

The main aim of this paper is to perform quantum mechanical calculations of electron-impact broadening of the transition $2 \mathrm{~s} 3 \mathrm{~s} \leftarrow 2 \mathrm{~s} 3 \mathrm{p}$ in Be-like ions, i.e., NIV, O V, F VI, and Ne VII. We also report new electron-impact broadening parameters for transitions in Be-like ions. We employ the impact approximation theory to obtain line broadening and shift parameters at various plasma conditions. In our numerical computations, Dirac Atomic R-matrix Codes (DARC) and General Purpose Relativistic Atomic Structure Package (GRASP0-10.10; Norrington 2009) are used to solve $(N+1)$-electron colliding systems so that the scattering matrices (reactance matrices) are obtained. The paper is organized as follows. Section 2 gives details of the methods used to calculate electron-impact broadening parameters. It also outlines the impact approximation and scattering matrix theory. Section 3 explains our strategy for constructing the atomic structure of each ion and selection of configurations. The comparison between experimental and theoretical data, accuracy of our results, final selected data of spectral line widths, the content and organization of the delivered tables are described in Sect. 4. Our conclusions are given in Sect. 5 .

\section{Theory and methods}

\subsection{Impact approximation theory}

In dense plasmas, spectral line broadening arises from perturbations by the constituents of the plasma (both ions and electrons). 
Ions have large masses than electron and always move much slowly, so in evaluating line broadening parameters it is often a good approximation to treat them as being stationary. The effect of stationary ions is equal to an average ion microfield, introduced by Hooper (1966). This microfield perturbs the radiating ions by Stark effect and leads to spectral line broadening, known as Stark broadening. Similarly, the high-speed electrons also perturb the emitting ions through collisions, and cause the interruption of spontaneous emission. This interruption will broaden the widths of emission lines, and is known as electron-impact broadening. The radiative transition of spontaneous emission between upper $\left(X_{b}\right)$ and lower state $\left(X_{a}\right)$ of radiating ion is written as:

$X_{b} \rightarrow X_{a}+\hbar \omega$.

The impact approximation theory was first formulated by Baranger (1958a-c) and later reviewed by Griem (1974). The theory considers a single binary collision between the perturber and the perturbed ion. During the time of electron collision with a radiating ion, the interaction between perturbing ions is ignored, and at a time each electron interacts only with one perturbed ion. Within impact approximation and the isolated line approximation, the line profile is Lorentzian, i.e.,

$\phi_{\omega}=\frac{w}{\pi} \frac{1}{\left(\omega-d-\omega_{0}\right)^{2}+w^{2}}$.

Where $\omega_{0}$ is the central line frequency, $d$ is the shift, and $2 w$ is the spectral line full width at half maximum. The width and shift of a spectral line are linearly proportional to electron density $N_{\mathrm{e}}$ and depend on the scattering matrix $\mathbf{S}$ for binary collisions. The final expression relating the shift and width of a spectral line could be obtained by averaging over Maxwell distribution of electron velocities. A simplified and compact expression has independently also been derived by Elabidi et al. 2004 in intermediate coupling scheme:

$w+\mathrm{i} d=\alpha N_{\mathrm{e}} \int_{0}^{\infty} T_{\mathrm{e}}^{-3 / 2} \exp (-\varepsilon / T) \Omega(\varepsilon) \mathrm{d} \varepsilon$.

Where parameter $\alpha=2.8674 \times 10^{-23} \mathrm{eV} \mathrm{cm}^{3}$. The units of electron density $N_{\mathrm{e}}$ is $\mathrm{cm}^{-3}$. Electron temperature $T_{\mathrm{e}}$ and colliding electron energy $\varepsilon$ are in Rydberg, and spectral line width $\mathrm{w}$ and shift $\mathrm{d}$ are in $\mathrm{eV}$. The electron collision strength $\Omega(\varepsilon)$ in jj coupling is defined by Peach (1996) (if intermediate coupling is considered, $\Omega(\varepsilon)$ can be found in the works of Elabidi et al. 2004; or Elabidi et al. 2008), i.e.,

$$
\begin{aligned}
\Omega(\varepsilon)= & \sum_{J_{i}^{T} J_{f}^{T} j j^{\prime} \ell \ell^{\prime}}(-1)^{J_{i}+J_{i^{\prime}}+2 J_{f}^{T}+j+j^{\prime}} \frac{1}{2}\left(2 J_{i}^{T}+1\right) \\
& \times\left(2 J_{f}^{T}+1\right)\left\{\begin{array}{ccc}
J_{f}^{T} & J_{i}^{T} & 1 \\
J_{i} & J_{f} & j
\end{array}\right\}\left\{\begin{array}{lll}
J_{f}^{T} & J_{i}^{T} & 1 \\
J_{i^{\prime}} & J_{f^{\prime}} & j^{\prime}
\end{array}\right\} \\
& \times \frac{1}{2}\left[\delta_{\ell \ell^{\prime}} \delta_{j j^{\prime}} \delta_{J_{i} J_{i^{\prime}}} \delta_{J_{f} J_{f^{\prime}}}-\mathbf{S}_{I}\left(J_{i^{\prime}} \ell^{\prime} j^{\prime} J_{i}^{T}, J_{i} \ell j J_{i}^{T}\right)\right. \\
& \left.\times \mathbf{S}_{F}^{*}\left(J_{f^{\prime}} \ell^{\prime} j^{\prime} J_{f}^{T}, J_{f} \ell j J_{f}^{T}\right)\right] .
\end{aligned}
$$

Where two $\{\ldots\}$ are the " $6 j$ " symbols. The upper and lower states of the transition are represented by the total angular momentum states $\left|J_{i} M_{i}\right\rangle$ and $\left|J_{f} M_{f}\right\rangle$ respectively, and $\mathbf{S}_{I}\left(J_{i^{\prime}} \ell^{\prime} j^{\prime} J_{i}^{T}, J_{i} \ell j J_{i}^{T}\right)$ is a scattering matrix element corresponding to an colliding electron scattered by the upper state $X_{b}$

$X_{b}+e(\varepsilon, \ell, j) \rightarrow X_{b}+e\left(\varepsilon, \ell^{\prime}, j^{\prime}\right)$.
Where $\ell, \ell^{\prime}$ and $j, j^{\prime}$ are the orbital and total angular momentum of colliding electrons before and after the collision with the target ion $X_{b}$ respectively. During the colliding process, the total energy $E_{I}=E_{b}+\varepsilon=E_{a}+\varepsilon+\omega$, total parity $\Pi$, total spin angular momentum $S^{T}$ and total angular momentum $J_{i}^{T}$ of $(N+1)$-electron colliding system are conserved. The $\mathbf{S}_{F}^{*}\left(J_{f^{\prime}} \ell^{\prime} j^{\prime} J_{f}^{T}, J_{f} \ell j J_{f}^{T}\right)$ is the conjugate matrix of a scattering matrix $\mathbf{S}$ corresponding to colliding process of the lower state $X_{a}$

$X_{a}+e(\varepsilon, \ell, j) \rightarrow X_{a}+e\left(\varepsilon, \ell^{\prime}, j^{\prime}\right)$.

In Eq. (6), the total energy $E_{F}=E_{a}+\varepsilon$, parity $\Pi$, total spin angular momentum $S^{T}$ and total angular momentum $J_{f}^{T}$ of $(N+1)$-electron colliding system are conserved. The above two scattering processes, described in Eqs. (5) and (6) will share the same total spin angular momentum $S^{T}$ and opposite parity $\Pi$ when only electric dipole transition is considered. This information is useful for the later numerical calculations.

\subsection{Scattering matrix}

The calculation of scattering matrices $S_{I}$ and $S_{F}$ is necessary to obtain electron collision strength $\Omega(\varepsilon)$. These two matrices are closely related to collision processes by Eqs. (5) and (6), which are $(N+1)$-electron colliding systems. Generally, a colliding system is constructed from $N$-electron target states $\Phi_{i}=\left|J_{i} M_{i}\right\rangle$ (possibly including pseudo states) and single particle wavefunctions $\phi_{i j}=|j m\rangle$ of the continuum electron. A state of such colliding system is well defined by the wavefunction in Berrington (1977) and Norrington (2009)

$\Psi_{k}=\sum_{i j} c_{i j k} \mathfrak{J}\left[\Phi_{i}, \phi_{i j}\right]+\sum_{\mathrm{m}} d_{m k} \theta_{\mathrm{m}}$

Where $\mathfrak{I}$ is an antisymmetric operator to account for the electron exchange, $j$ is the continuum basis function index for a particular $\kappa$ value $\left(\kappa=-\ell-1\right.$, if $\kappa<0$, else $\kappa=\ell$ ), and $\theta_{\mathrm{m}}$ is a capture state in which all of the $(N+1)$-electrons are bound.

The $(N+1)$-electron colliding system, defined by Eqs. (5) or (6), is solved by using DARC to obtain a reactance $\mathbf{K}$-matrix which is real symmetric matrix. With K-matrix, scattering matrix $\mathbf{S}$ is defined as:

$\mathbf{S}=\frac{1+\mathrm{i} \mathbf{K}}{1-\mathrm{iK}}$

From Eq. (8), $\mathbf{S}$ is an unitary matrix, and its real part and imaginary part are written out as

$\operatorname{Re} \mathbf{S}=\frac{1-\mathbf{K}^{2}}{\left(1+\mathbf{K}^{2}\right)}$.
$\operatorname{Im} \mathbf{S}=\frac{2 \mathbf{K}}{\left(1+\mathbf{K}^{2}\right)}$.

These two expressions are directly used in our numerical calculations.

\section{Numerical calculations in detail}

To obtain electron collision strength $\Omega(\varepsilon)$, we solved the colliding system which consists of an $N$-electron target ion and a colliding electron. The states of an $N$-electron target in DARC calculations are prepared first by a fully relativistic atomic structure code called GRASP0-10.10 as explained in the next section. 


\subsection{Atomic structure}

In our calculations, the atomic structure data of four Be-like ions from NIV to Ne VII are obtained by using the multiconfiguration Dirac-Fock (MCDF) atomic structure program GRASP0-10.10 developed by Dyall et al. (1989) and Parpia et al. (1996) included in DARC. The original GRASP0-10.10 have been partly improved by us according to our previous works (Duan et al. 2008; Bari et al. 2011) so that all the following atomic structure data can be calculated by extended average-level (EAL) mode. To calculate the atomic structure of the four Be-like ions, i.e., NIV, O V, F VI and Ne VII, we have selected the following electronic configurations: $1 \mathrm{~s}^{2} 2 \mathrm{~s}^{2}$, $1 s^{2} 2 s 2 p, 1 s^{2} 2 s 3 s, 1 s^{2} 2 s 3 p, 1 s^{2} 2 s 3 d, 1 s^{2} 2 s 4 s, 1 s^{2} 2 s 4 p, 1 s^{2} 2 s 5 s$, and $1 \mathrm{~s}^{2} 2 \mathrm{~s} 5 \mathrm{p}$. With the selection of these configurations, it is easy to obtain the electronic orbitals, the energy levels and states of target ions. These atomic data are incorporated as input in solving $(N+1)$-electron colliding system by DARC.

\subsection{From $\mathbf{K}$ to $\mathbf{S}$}

With the atomic structure data of target ions, the $(N+1)$-electron colliding systems of Eqs. (5) and (6) are solved by DARC, and their $\mathbf{K}$ matrices and quantum numbers of angular momentum are also obtained. Then $\mathbf{K}$ matrix is used to calculate the real and imaginary parts of scattering matrix $\mathbf{S}$ with Eqs. (9). The transformation from $\mathbf{K}$ to $\mathbf{S}$ is performed by a code developed by us.

\section{Numerical results and discussion}

In this paper, the electron-impact broadening widths of the transition arising from the $2 \mathrm{~s} 3 \mathrm{~s} \leftarrow 2 \mathrm{~s} 3 \mathrm{p}$ configuration in Be-like ions NIV, O V, FVI and Ne VII are calculated. In these calculations, we have used the GRASP0-10.10 package included in DARC to provide the fine-structure wavefunctions, energy levels, wavelengths, and transition rates. We obtained the electron impact collision strengths by DARC. For those configurations, each target ion has 27 bound states. All of these target states are selected as target basis in our calculations. To generate and construct the $(N+1)$-electron colliding systems defined in Eqs. (5) and (6), two parameters of the colliding electron are defined by specific boundary limits i.e., the quantum number of orbital angular momentum is $\ell \leq 10$ and the total number of continuum basis function for a given $\kappa$ is 14. The electron collision strength $\Omega(\varepsilon)$ defined in Eq. (4) is a function of colliding electron energy $\varepsilon$ and is computed in an increasing energy sequence with an energy step of $\Delta \varepsilon=0.02$ Rydberg by the same procedure. To realize and confirm our numerical calculations, it is necessary for us to study the convergence of collision strength $\Omega(\varepsilon)$ with both the orbital angular momentum $\ell$ and the colliding electron energy $\varepsilon$. In Fig. 1 the maximum $\ell$ of the colliding electron in Eq. (4) is limited by 10 or 15 , and $\Omega(\varepsilon)$ are present as a function $\varepsilon$ in transition ${ }^{3} \mathrm{~S}_{1} \leftarrow{ }^{3} \mathrm{P}_{1}$ in N IV ion. It can be seen that $\Omega(\varepsilon)$ converges with the orbital angular momentum $\ell$ if $\ell$ is large enough, and that it also decreases with the colliding electron energy $\varepsilon$. Thus the electron impact broadening in Eq. (3) is numerically calculated by the trapezoidal integration technique. The full width at half maximum ( $F W H M$ $W=2 w)$ for transitions $2 \mathrm{~s} 3 \mathrm{~s} \leftarrow 2 \mathrm{~s} 3 \mathrm{p}$ for various electron temperature $T_{\mathrm{e}}$ and electron density $N_{\mathrm{e}}$ are given in Tables 1-7. The full quantum mechanical calculations of line widths $w_{2}$ and its simplified calculations $w_{1}$ by Elabidi et al. (2008) are also listed in Tables 1-7 for comparison purposes. These $w_{2}$ and $w_{1}$

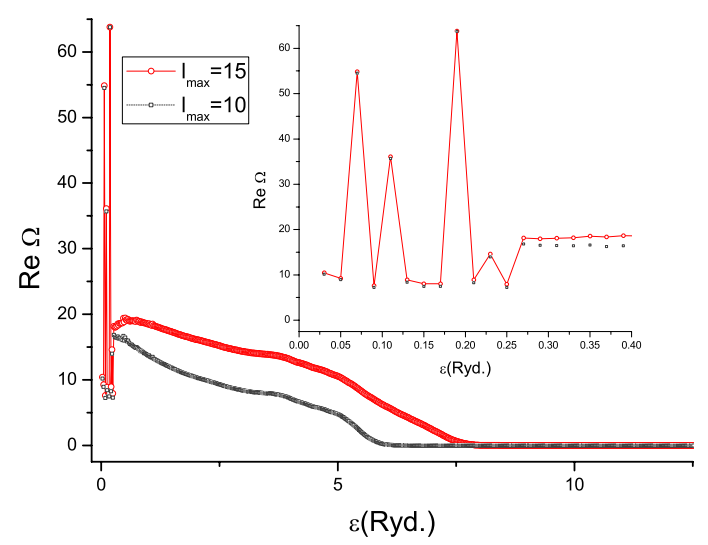

Fig. 1. Real part of the collision strength as a function of the colliding electron energy for two values of the maximum angular momentum $l_{\max }$. Squares: $l_{\max }=10$. Circles: $l_{\max }=15$.

were calculated in an intermediate coupling framework by including the fine-structure effects and relativistic corrections resulting from breakdown of the LS coupling approximation for the target ions. A comparison between these calculations and experimental results has proven to be a good agreement for the first time for highly charged ions. The line widths $w_{\mathrm{c}}$ calculated by Ralchenko et al. (2001) with convergent close-coupling methods are also presented. These authors find (i) that calculated widths are generally smaller than both those measured and most of theoretical line widths from semiclassical calculations; and (ii) that the deviation between experiment and theory monotonically increases along the isoelectronic sequence, reaching a factor of 2 for Ne VII.

We performed calculations for each ion for the temperature values for which the experimental data are available. Principally, all the corresponding experimental results cited in the present work have been published by two groups. The first group (Blagojević et al. 1999, 2000) used a low-pressure pulsed arc and the second group (Wrubel et al. 1996, 1998) used a gas-liner pinch. The accuracy of the experimental line widths is generally given by Konjević et al. (2002) except for the few cases where other references have been mentioned. For $2 \mathrm{~s} 3 \mathrm{~s}{ }^{1} \mathrm{~S} \leftarrow$ $2 \mathrm{~s} 3 \mathrm{p}^{1} \mathrm{P}$ transitions in F VI ions, we compared our results with $w_{1}$ of Elabidi et al. (2008) as there are no experimental results available yet.

To assess the quality of our calculations, we performed systematic comparisons with earlier experimental and theoretical data. Our results in Tables 1-7 agree well with both the experimental results and the previous theoretical calculations. Our results for the triplet transitions of NIV, for both the singlet and triplet transitions of $\mathrm{OV}$, and singlet transitions of Ne VII are found to be in excellent agreement with the corresponding experimental results. However, the line widths for triplet transitions of Ne VII revealed a significant difference between our calculations and the corresponding measurements (see Table 6). Our calculated width of the transition line ${ }^{3} \mathrm{~S}_{1} \leftarrow{ }^{3} \mathrm{P}_{1}$ at vacuum wavelength $1992.06 \AA$ is about $0.308 \AA$, while the corresponding measurement is about $0.45 \AA$ and the difference amounts to $32 \%$. We summarize our results by examining them in the following section by presenting line widths $W=2 w(F W H M)$ of singlet and triplet transitions $2 \mathrm{~s} 3 \mathrm{~s} \leftarrow 2 \mathrm{~s} 3 \mathrm{p}$ in four Be-like ions from N IV to Ne VII:

Line widths of NIV. In Tables 1 and 2, we present our results for the line widths of singlet and triplet transitions $2 \mathrm{~s} 3 \mathrm{~s} \leftarrow 2 \mathrm{~s} 3 \mathrm{p}$ 
Table 1. Electron-impact widths $W$ (FWHM) for the NIV $2 \mathrm{~s} 3 \mathrm{~s}{ }^{3} \mathrm{~S} \leftarrow 2 \mathrm{~s} 3 \mathrm{p}{ }^{3} \mathrm{P}$ transitions at various electron temperatures $T_{\mathrm{e}}$ and electron densities $N_{\mathrm{e}}$.

\begin{tabular}{|c|c|c|c|c|c|c|c|}
\hline $\begin{array}{l}\text { N IV } \\
\text { Transition }\end{array}$ & $\begin{array}{c}T_{\mathrm{e}} \\
\left(10^{4} \mathrm{~K}\right) \\
\end{array}$ & $\begin{array}{c}N_{\mathrm{e}} \\
\left(10^{17} \mathrm{~cm}^{-3}\right) \\
\end{array}$ & $\begin{array}{l}W \\
(\AA) \\
\end{array}$ & $\begin{array}{l}w_{1} \\
(\AA) \\
\end{array}$ & $\begin{array}{l}w_{2} \\
(\AA) \\
\end{array}$ & $\begin{array}{l}w_{\mathrm{m}} \\
(\AA) \\
\end{array}$ & $\begin{array}{l}w_{\mathrm{c}} \\
(\AA)\end{array}$ \\
\hline $\begin{array}{l}{ }^{3} \mathrm{~S}_{1} \leftarrow{ }^{3} \mathrm{P}_{2} \\
(3478.72 \AA)\end{array}$ & $\begin{array}{c}5.4 \\
7.24 \\
7.83 \\
8.01 \\
8.07 \\
8.12 \\
8.23 \\
9.63 \\
9.86 \\
10.1 \\
10.44 \\
\end{array}$ & $\begin{array}{c}2.8 \\
0.86 \\
0.99 \\
1.13 \\
1.21 \\
5.80 \\
1.33 \\
8.1 \\
15.8 \\
19.4 \\
10.6 \\
\end{array}$ & $\begin{array}{c}0.400 \\
0.110 \\
0.122 \\
0.138 \\
0.147 \\
0.703 \\
0.160 \\
0.909 \\
1.75 \\
2.213 \\
1.15 \\
\end{array}$ & $\begin{array}{c}0.381 \\
0.116 \\
0.132 \\
0.151 \\
0.161 \\
0.773 \\
0.177 \\
1.06 \\
2.05 \\
2.51 \\
1.36 \\
\end{array}$ & $\begin{array}{c}0.555 \\
0.170 \\
0.194 \\
0.221 \\
0.237 \\
1.13 \\
0.26 \\
1.55 \\
3.02 \\
3.69 \\
2.01 \\
\end{array}$ & $\begin{array}{c}0.410^{c} \\
0.122^{a} \\
0.136^{a} \\
0.148^{a} \\
0.153^{a} \\
0.84^{b} \\
0.172^{a} \\
1.11^{b} \\
1.46^{b} \\
1.72^{b} \\
1.11^{b} \\
\end{array}$ & $\begin{array}{l}0.246 \\
0.070 \\
0.080 \\
0.090 \\
0.094 \\
0.452 \\
0.102 \\
0.613 \\
1.168 \\
1.421 \\
0.776 \\
\end{array}$ \\
\hline $\begin{array}{l}{ }^{3} \mathrm{~S}_{1} \leftarrow{ }^{3} \mathrm{P}_{1} \\
(3483.00 \AA)\end{array}$ & $\begin{array}{c}5.4 \\
7.24 \\
7.83 \\
8.01 \\
8.07 \\
8.12 \\
8.23 \\
9.63 \\
9.86 \\
10.1 \\
10.44 \\
\end{array}$ & $\begin{array}{c}2.8 \\
0.86 \\
099 \\
1.13 \\
1.21 \\
5.8 \\
1.33 \\
8.1 \\
15.8 \\
19.4 \\
10.6 \\
\end{array}$ & $\begin{array}{c}0.403 \\
0.110 \\
0.123 \\
0.139 \\
0.148 \\
0.708 \\
0.161 \\
0.916 \\
1.77 \\
2.15 \\
1.15 \\
\end{array}$ & $\begin{array}{c}0.383 \\
0.117 \\
0.133 \\
0.152 \\
0.163 \\
0.778 \\
0.178 \\
1.06 \\
2.08 \\
2.53 \\
1.38 \\
\end{array}$ & $\begin{array}{c}0.564 \\
0.172 \\
0.197 \\
0.225 \\
0.240 \\
1.15 \\
0.264 \\
1.58 \\
3.07 \\
3.75 \\
2.04 \\
\end{array}$ & $\begin{array}{c}0.330^{c} \\
0.122^{a} \\
0.136^{a} \\
0.150^{a} \\
0.150^{a} \\
0.84^{b} \\
0.174 \\
1.10^{b} \\
1.46^{b} \\
1.72^{b} \\
1.11^{b} \\
\end{array}$ & $\begin{array}{l}0.246 \\
0.070 \\
0.080 \\
0.090 \\
0.095 \\
0.449 \\
0.102 \\
0.604 \\
1.168 \\
1.433 \\
0.776 \\
\end{array}$ \\
\hline $\begin{array}{l}{ }^{3} \mathrm{~S}_{1} \leftarrow{ }^{3} \mathrm{P}_{0} \\
(3484.93 \AA)\end{array}$ & $\begin{array}{c}5.4 \\
7.24 \\
7.83 \\
8.01 \\
8.07 \\
8.12 \\
8.23 \\
9.63 \\
9.86 \\
10.1 \\
10.44\end{array}$ & $\begin{array}{c}2.8 \\
0.86 \\
0.99 \\
1.13 \\
1.21 \\
5.8 \\
1.33 \\
8.1 \\
15.8 \\
19.4 \\
10.6\end{array}$ & $\begin{array}{c}0.400 \\
0.110 \\
0.122 \\
0.138 \\
0.147 \\
0.705 \\
0.161 \\
0.912 \\
1.76 \\
2.14 \\
1.15\end{array}$ & $\begin{array}{l}0.385 \\
0.117 \\
0.134 \\
0.153 \\
\\
0.782 \\
\\
1.07 \\
2.08 \\
2.54 \\
1.38\end{array}$ & $\begin{array}{l}0.571 \\
0.174 \\
0.199 \\
0.227 \\
\\
1.16 \\
\\
1.59 \\
3.09 \\
3.78 \\
2.06\end{array}$ & $\begin{array}{c}0.400^{c} \\
0.122^{a} \\
0.146^{a} \\
0.146^{a} \\
\\
0.84^{b} \\
\\
1.10^{b} \\
1.46^{b} \\
1.72^{b} \\
1.11^{b}\end{array}$ & $\begin{array}{l}0.245 \\
0.070 \\
0.080 \\
0.090\end{array}$ \\
\hline
\end{tabular}

Notes. Vacuum wavelengths given in parentheses for each transition, $W$ presents calculations, $w_{2}$ full quantum mechanical calculations, $w_{1}$ simplified calculations of Elabidi et al. (2008), $w_{\mathrm{m}}$ experimental widths, $w_{\mathrm{c}}$ the quantum calculations of Ralchenko et al. (2001) (same notations in the following tables); ${ }^{(a)}{ }^{(b)}$, and ${ }^{(c)}$ represent for experiments Blagojević et al. (1999), Wrubel et al. (1998), and Milosavljević \& Djenize (1998), respectively.

Table 2. Electron-impact widths $W$ (FWHM) for $2 \mathrm{~s} 3 \mathrm{~s}{ }^{1} \mathrm{~S}_{1} \leftarrow 2 \mathrm{~s} 3 \mathrm{p}{ }^{1} \mathrm{P}_{1}$ transitions of N IV, where the line widths are listed as a function of electron temperature $T_{\mathrm{e}}$ under electron density $N_{\mathrm{e}}=10^{18} \mathrm{~cm}^{-3}$.

\begin{tabular}{l|cccccc}
\hline \hline $\begin{array}{l}\text { NIV } \\
\text { Transition }\end{array}$ & $\begin{array}{c}T_{\mathrm{e}} \\
\left(10^{4} \mathrm{~K}\right)\end{array}$ & $\begin{array}{c}W \\
(\AA)\end{array}$ & $\begin{array}{c}w_{1} \\
(\AA)\end{array}$ & $\begin{array}{c}w_{2} \\
(\AA)\end{array}$ & $\begin{array}{c}w_{\mathrm{m}} \\
(\AA)\end{array}$ & $\begin{array}{c}w_{\mathrm{c}} \\
(\AA)\end{array}$ \\
\hline${ }^{1} \mathrm{~S}_{0} \leftarrow{ }^{1} \mathrm{P}_{1}$ & 2.320890 & 4.80 & 4.41 & 6.27 & & \\
$(6380.75 \AA)$ & 4.641779 & 3.47 & 4.55 & 6.59 & & \\
& 6.962669 & 2.77 & 4.46 & 6.49 & & \\
& 7.190000 & 2.72 & 4.45 & 6.46 & 3.89 & 2.82 \\
& 8.120000 & 2.53 & 4.37 & 6.37 & 3.76 & 2.72 \\
& 9.283559 & 2.34 & 4.27 & 6.24 & & \\
& 11.60445 & 2.04 & 4.07 & 5.95 & & \\
& 13.92534 & 1.81 & 3.87 & 5.66 & & \\
& 16.24623 & 1.63 & 3.69 & 5.40 & & \\
& 18.56712 & 1.49 & 3.51 & 5.15 & & \\
& 20.88801 & 1.37 & 3.37 & 4.94 & & \\
& 23.20890 & 1.27 & 3.23 & 4.74 & & \\
\hline
\end{tabular}

Notes. Experimental widths $w_{\mathrm{m}}$ are cited from Wrubel et al. (1998). 
Table 3. Electron-impact widths $W$ (FWHM) for the O V $2 \mathrm{~s} 3 \mathrm{~s}^{3} \mathrm{~S} \leftarrow 2 \mathrm{~s} 3 \mathrm{p}^{3} \mathrm{P}$ transitions.

\begin{tabular}{l|ccccccc}
\hline \hline $\begin{array}{l}\text { O V } \\
\text { Transition }\end{array}$ & $\begin{array}{c}T_{\mathrm{e}} \\
\left(10^{4} \mathrm{~K}\right)\end{array}$ & $\begin{array}{c}N_{\mathrm{e}} \\
\left(10^{17} \mathrm{~cm}^{-3}\right)\end{array}$ & $\begin{array}{c}W \\
(\AA)\end{array}$ & $\begin{array}{c}w_{1} \\
(\AA)\end{array}$ & $\begin{array}{c}w_{2} \\
(\AA)\end{array}$ & $\begin{array}{c}w_{\mathrm{m}} \\
(\AA)\end{array}$ & $\begin{array}{c}w_{\mathrm{c}} \\
(\AA)\end{array}$ \\
\hline${ }^{3} \mathrm{~S}_{1} \leftarrow{ }^{3} \mathrm{P}_{1}$ & 5.46 & 0.95 & 0.0573 & 0.054 & 0.079 & 0.077 & 0.0360 \\
$(2786.99 \AA)$ & 6.19 & 1.38 & 0.0807 & 0.079 & 0.116 & 0.102 & 0.051 \\
& 6.55 & 1.09 & 0.0628 & 0.062 & 0.092 & 0.073 & 0.0399 \\
& 7.97 & 1.41 & 0.0764 & 0.080 & 0.118 & 0.100 & 0.050 \\
& 8.87 & 1.57 & 0.0820 & 0.088 & 0.131 & 0.105 & 0.0541 \\
& 9.16 & 1.68 & 0.0867 & 0.094 & 0.140 & 0.102 & 0.0570 \\
& 9.33 & 1.54 & 0.0790 & 0.086 & 0.128 & 0.105 & 0.0530 \\
\hline${ }^{3} \mathrm{~S}_{1} \leftarrow{ }^{3} \mathrm{P}_{0}$ & 6.19 & 1.38 & 0.0819 & 0.079 & 0.117 & 0.098 & 0.0510 \\
$(3484.93 \AA)$ & 7.97 & 1.42 & 0.0780 & 0.081 & 0.121 & 0.098 & 0.050 \\
& 8.87 & 1.57 & 0.0830 & 0.088 & 0.133 & 0.105 & 0.0541 \\
& 9.16 & 1.68 & 0.0878 & 0.094 & 0.142 & 0.108 & 0.0571 \\
\hline
\end{tabular}

Notes. $w_{\mathrm{m}}$ from Blagojević et al. (1999).

Table 4. Electron-impact widths $W$ (FWHM) for the O V $2 \mathrm{~s} 3 \mathrm{~s}{ }^{1} \mathrm{~S} \leftarrow 2 \mathrm{~s} 3 \mathrm{p}{ }^{1} \mathrm{P}$ transitions.

\begin{tabular}{l|ccccccc}
\hline \hline $\begin{array}{l}\mathrm{OV} \\
\text { Transition }\end{array}$ & $\begin{array}{c}T_{\mathrm{e}} \\
\left(10^{4} \mathrm{~K}\right)\end{array}$ & $\begin{array}{c}N_{\mathrm{e}} \\
\left(10^{17} \mathrm{~cm}^{-3}\right)\end{array}$ & $\begin{array}{c}W \\
(\AA)\end{array}$ & $\begin{array}{c}w_{1} \\
(\AA)\end{array}$ & $\begin{array}{c}w_{2} \\
(\AA)\end{array}$ & $\begin{array}{c}w_{\mathrm{m}} \\
(\AA)\end{array}$ & $\begin{array}{c}w_{\mathrm{c}} \\
(\AA)\end{array}$ \\
\hline${ }^{1} \mathrm{~S}_{1} \leftarrow{ }^{1} \mathrm{P}_{1}$ & 9.05 & 10.8 & 2.22 & 1.94 & 2.89 & 1.85 & 1.12 \\
$(5114.06 \AA)$ & 10.8 & 12.0 & 2.25 & 2.10 & 3.13 & 2.01 & 1.21 \\
& 16.7 & 17.2 & 2.54 & 2.74 & 4.08 & 2.16 & 1.57 \\
& 21.7 & 23.6 & 2.97 & 3.48 & 5.16 & 2.85 & 2.04 \\
\hline
\end{tabular}

Notes. $w_{\mathrm{m}}$ from Wrubel et al. (1998).

Table 5. Electron-impact widths $W$ (FWHM) for the F VI $2 \mathrm{~s} 3 \mathrm{~s}{ }^{1} \mathrm{~S} \leftarrow$ $2 \mathrm{~s} 3 \mathrm{p}{ }^{1} \mathrm{P}$ transitions listed as a function of electron temperature $T_{\mathrm{e}}$ under electron density $N_{\mathrm{e}}=10^{18} \mathrm{~cm}^{-3}$.

\begin{tabular}{ccc}
\hline \hline $\mathrm{F} \mathrm{VI} 2 \mathrm{~s} 3 \mathrm{~s}{ }^{1} \mathrm{~S}_{0} \leftarrow 2 \mathrm{~s} 3 \mathrm{p}^{1} \mathrm{P}_{1}$ & $(6830.75 \AA)$ & $N_{\mathrm{e}}=10^{18} \mathrm{~cm}^{-3}$ \\
$T_{\mathrm{e}}$ & $W$ & $w_{1}$ \\
$\left(10^{4} \mathrm{~K}\right)$ & $(\AA)$ & $(\AA)$ \\
\hline 2.320890 & 10.376 & 0.757 \\
4.641779 & 5.981 & 0.869 \\
6.962669 & 4.318 & 0.889 \\
9.283559 & 3.441 & 0.881 \\
11.60445 & 2.893 & 0.861 \\
13.92534 & 2.514 & 0.836 \\
16.24623 & 2.235 & 0.810 \\
18.56712 & 2.019 & 0.782 \\
20.88801 & 1.846 & 0.757 \\
23.20890 & 1.704 & 0.734 \\
\hline
\end{tabular}

of N IV ion for various electron temperatures and densities along with the experimental results (Blagojević et al. 1999, 2000; Wrubel et al. 1996, 1998). The previous theoretical calculations $w_{\mathrm{c}}$ of Ralchenko et al. 2001), the full quantum mechanical calculations $w_{2}$ and its simplified calculations $w_{1}$ of Elabidi et al. (2008) are also listed in Tables 1 and 2. The experimental measurements $w_{\mathrm{m}}$ give singlet and triplet transitions $2 \mathrm{~s} 3 \mathrm{~s} \leftarrow 2 \mathrm{~s} 3 \mathrm{p}$ line widths of N IV ions at different electron temperatures. The measurements by Milosavljević \& Djenize (1998) were carried out for electron temperature $T_{\mathrm{e}}=5.4 \times 10^{4} \mathrm{~K}$, while Blagojević et al. (1999) considered some lower temperatures, and Wrubel et al. (1998) measured at higher temperatures. From Table 1, our results are in good agreement with the experimental values. In detail, most of the other spectral line widths are in good agreement with $w_{\mathrm{m}}$ within $5 \%$. The difference for the majority of the spectral lines amounts to $10 \%$ and the most significant difference reaches to $18 \%$. Additionally, there are a few cases where the difference between our results and existing experimental values is large. For example, in the transition ${ }^{3} \mathrm{~S}_{1} \leftarrow{ }^{3} \mathrm{P}_{2}$ under plasma condition $T_{\mathrm{e}}=9.63 \times 10^{4} \mathrm{~K}$ and $N_{\mathrm{e}}=8.1 \times 10^{17} \mathrm{~cm}^{-3}$, the difference in the line width reaches to $18 \%$. Similarly, the transitions ${ }^{3} \mathrm{~S}_{1} \leftarrow{ }^{3} \mathrm{P}_{1}$ and ${ }^{3} \mathrm{~S}_{1} \leftarrow{ }^{3} \mathrm{P}_{0}$ in Table 1 have two and four exceptional cases, respectively, which our results show as large deviations from experimental values. For singlet transitions listed in Table 2, it can be seen that the discrepancies between $W$ and two experimental results $w_{\mathrm{m}}$ cited by Bochum Group (Wrubel et al. 1996, 1998) are within 33\%. Our results are less than experimental $w_{\mathrm{m}}$ in contrast to $w_{1}$. When compared with $w_{\mathrm{m}}$, our results are better than $w_{2}$, but worse than $w_{1}$. Overall, our results $W$ for triplet transitions are always better than those of singlet transitions.

Line widths of $O V$. In Tables 3 and 4, we compare our theoretical calculations of line widths for the singlet and triplet transitions $2 \mathrm{~s} 3 \mathrm{~s} \leftarrow 2 \mathrm{~s} 3 \mathrm{p}$ of $\mathrm{O} \mathrm{V}$ ion for various electron temperatures and densities. We also note that the measured widths are determined with an error of $23 \%$ : the error of the temperature determination is $18-27 \%$ and $15 \%$ for the density determination (Elabidi et al. 2008). In triplet transitions in $\mathrm{O}$ V, our results are consistent with $w_{1}$ and agree with each other, but $w_{2}$ are closer to the experimental values when compared to our calculations. However, in singlet transitions, $w_{1}$ is the best in two first lines of the table, and our results are the best in two last lines, while $w_{2}$ is the worst. Most of our line width results are within an error of $15 \%$, and the maximum error is about $25 \%$ in comparison to the experimental results.

Line widths of FVI. In Table 5, we list our results $W$ as a function of electron temperature under an electron density 
Table 6. Electron-impact widths $W$ (FWHM) for the Ne VII $2 \mathrm{~s} 3 \mathrm{~s}^{3} \mathrm{~S} \leftarrow 2 \mathrm{~s} 3 \mathrm{p}^{3} \mathrm{P}$ transitions under electron density of $N_{\mathrm{e}}=3 \times 10^{18} \mathrm{~cm}^{-3}$.

\begin{tabular}{ccccc}
\hline \hline Ne VII & $T_{\mathrm{e}}=2.38 \times 10^{5} \mathrm{~K}$ & \multicolumn{3}{c}{$N_{\mathrm{e}}=3 \times 10^{18} \mathrm{~cm}^{-3}$} \\
Transition & $W$ & $w_{1}$ & $w_{\mathrm{m}}$ & $w_{\mathrm{c}}$ \\
$(\AA)$ & $(\AA)$ & $(\AA)$ & $(\AA)$ & $(\AA)$ \\
\hline${ }^{3} \mathrm{~S}_{1} \leftarrow{ }^{3} \mathrm{P}_{2}(1981.97 \AA)$ & 0.309 & 0.378 & $0.45^{a}$ & 0.230 \\
${ }^{3} \mathrm{~S}_{1} \leftarrow{ }^{3} \mathrm{P}_{1}(1992.06 \AA)$ & 0.308 & 0.383 & $0.45^{b}$ & 0.230 \\
${ }^{3} \mathrm{~S}_{1} \leftarrow{ }^{3} \mathrm{P}_{0}(1997.35 \AA)$ & 0.309 & 0.391 & $0.45^{a}$ & 0.230 \\
\hline
\end{tabular}

Notes. ${ }^{(a)}$ and ${ }^{(b)}$ selected from Wrubel et al. (1996) and Wrubel et al. (1998) respectively; $w_{\mathrm{c}}$ : the results of Ralchenko et al. (1999).

Table 7. Electron-impact widths $W$ (FWHM) for the Ne VII $2 \mathrm{~s} 3 \mathrm{~s}^{1} \mathrm{~S} \leftarrow$ $2 \mathrm{~s} 3 \mathrm{p}^{1} \mathrm{P}$ transitions under electron density of $N_{\mathrm{e}}=3.5 \times 10^{18} \mathrm{~cm}^{-3}$ and electron temperature $T_{\mathrm{e}}=2.205 \times 10^{5} \mathrm{~K}$.

\begin{tabular}{lccccc}
\hline \hline Ne VII & \multicolumn{3}{c}{$T_{\mathrm{e}}=2.205 \times 10^{5} \mathrm{~K}$} & \multicolumn{3}{c}{$N_{\mathrm{e}}=3.5 \times 10^{18} \mathrm{~cm}^{-3}$} \\
Transition & $W$ & $w_{1}$ & $w_{2}$ & $w_{\mathrm{m}}$ & $w_{\mathrm{c}}$ \\
$(\AA)$ & $(\AA)$ & $(\AA)$ & $(\AA)$ & $(\AA)$ & $(\AA)$ \\
\hline${ }^{1} \mathrm{~S}_{0} \leftarrow{ }^{1} \mathrm{P}_{1}$ & & & & & \\
$(3643.60 \AA)$ & 1.32 & 1.17 & 1.5 & 1.70 & 1.00 \\
\hline
\end{tabular}

Notes. The experimental result is selected from Wrubel et al. (1998), and $w_{\mathrm{c}}$ is result of Ralchenko et al. (1999).

$N_{\mathrm{e}}=10^{18} \mathrm{~cm}^{-3}$. There are no experimental results available for F VI ${ }^{1} \mathrm{~S}_{0} \leftarrow{ }^{1} \mathrm{P}_{1}^{\circ}$ line widths. Only the quantum mechanical calculations $w_{1}$ of Elabidi et al. (2008) are listed in Table 5 for comparison purposes. Our calculations show large differences from $w_{1}$ results. Regarding this discrepancy between the quantum mechanical calculation $w_{1}$ and our theoretical calculations, the reader may wonder about the cause of such a difference. At present, we cannot understand this discrepancy. The reasons of this discrepancy need to be investigated both experimentally and theoretically in future.

Line widths of Ne VII. In Table 6, we report our results for the triplet transitions of Ne VII along with the experimental line widths measured by the Bochum group (Wrubel et al. 1996, 1998) at electron density $N_{\mathrm{e}}=3 \times 10^{18} \mathrm{~cm}^{-3}$ and electron temperature $T_{\mathrm{e}}=2.38 \times 10^{5} \mathrm{~K}$. A comparison between our results and the measurements yields a maximum difference of $32 \%$. Our results are in good agreement with the theoretical calculations $w_{1}$.

In Table 7, we present the line widths for singlet transition of Ne VII ion at electron density $N_{\mathrm{e}}=3.5 \times 10^{18} \mathrm{~cm}^{-3}$ and electron temperature $T_{\mathrm{e}}=2.205 \times 10^{5} \mathrm{~K}$. A comparison between $W$ and $w_{\mathrm{m}}$ (Wrubel et al. 1998) shows that the difference is within $22 \%$. It indicates that our results $W$ for line widths of Ne VII are in good agreement with $w_{\mathrm{m}}$ and are more accurate than $w_{1}$ but less accurate than $w_{2}$.

\section{Conclusions}

In the present work, a new set of electron-impact broadening of the singlet and triplet transition $2 \mathrm{~s} 3 \mathrm{~s} \leftarrow 2 \mathrm{~s} 3 \mathrm{p}$ in four Be-like ions from NIV to Ne VII have been obtained by solving the $(N+1)$-electron colliding systems of Be-like ions with DARC on the basis of relativistic quantum mechanical approach. Spectral line widths of triplet transitions of N IV ion are in best agreement with experimental values. A maximum discrepancy of $32 \%$ appears between $\mathrm{W}$ and $w_{\mathrm{m}}$ for triplet transitions of $\mathrm{Ne}$ VII ion at electron density $N_{\mathrm{e}}=3 \times 10^{18} \mathrm{~cm}^{-3}$ and electron temperature $T_{\mathrm{e}}=2.38 \times 10^{5} \mathrm{~K}$, which can be considered as tolerable agreement with respect to the accuracy of the experiment. Overall, one can conclude that our results for all defined temperatures agree with the experimental values within $10 \%, 20 \%$, and $30 \%$ for N IV, O V, and Ne VII respectively. For singlet transitions in F VI ion (see Table 5), our results are quite different from $w_{1}$, which needs clarification in future studies. Our calculations further support the argument of good agreement between quantum mechanical calculations and experiments for line widths of $\Delta n=0$ transitions in highly charged ions in accordance with the conclusion of Elabidi et al. (2008). Our present calculations will be used for plasma diagnostics.

Acknowledgements. This work was supported by the National Natural Science Foundation of China (Grant No. 11275029) and the Foundation for Development of Science and Technology of Chinese Academy of Engineering Physics (Grant Nos. 2011A0102007 and 2009A0102006).

\section{References}

Baranger, M. 1958a, Phys. Rev., 111, 481

Baranger, M. 1958b, Phys. Rev., 111, 494

Baranger, M. 1958c, Phys. Rev., 112, 855

Bari, M. A., Salahuddin, M., Duan, B., Nasim, M. H., \& Hussain, S. A. 2011, J. Phys. B, 44, 225004

Berrington, K. A., Burke, P. G., Dufton, P. L., \& Kington, A. E. 1977, J. Phys. $\mathrm{B}, 8,1465$

Blagojević, B., Popović, M. V., Konjević, N., \& Dimitrijević, M. S. 1999, J. Quant. Spectr. Radiat. Trans., 61, 361

Blagojević, B., Popović, M. V., \& Konjević, N. 2000, J. Quant. Spectr. Radiat. Trans., 67, 9

Dimitrijević, M. S., \& Konjević, N. 1980, J. Quant. Spectr. Radiat. Trans., 24, 451

Duan, B., Bari, M. A., Zhong, J. Y., et al. 2008, A\&A, 488, 1155

Dyall, K. G., Grant, I. P., Johnson, C. T., Parpia, F. A., \& Plummer, E. P. 1989, Comput. Phys. Commun., 55, 425

Elabidi, H., Ben Nessib, N., \& Sahal-Bréchot, S. 2004, J. Phys. B, 37, 63

Elabidi, H., Ben Nessib, N., Cornille, M., Dubau, J., \& Sahal-Bréchot, S. 2008, J. Phys. B, 41, 025702

Griem, H. R. 1974, Spectral Line Broadening by Plasma (NewYork, USA: Academic press)

Hooper, C. F. Jr. 1966, Phys. Rev., 149, 77

Konjević, N., Lesage, A., Fuhr, J. R., \& Wiese, W. L. 2002, J. Phys. Chem. Ref. Data, 31, 819

Milosavljević, V., \& Djenize, S. 1998, A\&AS, 128, 179

Norrington, P. 2009, DARC manual, http://www . am.qub.ac.uk/users/p. norrington

Ralchenko, Y., Griem, H. R., Bray, I., \& Fursa, D. V. 1999, Phys. Rev. A, 59, 1890

Ralchenko, Y., Griem, H. R., Bray, I., \& Fursa, D. V. 2001, J. Quant. Spectr. Radiat. Trans., 71, 595

Seaton, M. J. 1987, J. Phys. B, 20, 6431

Parpia, F. A., Froese Fischer, C., \& Grant, I. P. 1996, Comput. Phys. Commun., 94, 249

Peach, G. 1996, in Atomic, Molecular \& Optical Physics Handbook, ed. G. W. F. Drake (New York: AIP Press), 669

Wrubel, Th., Glenzer, S., Bücher, S., Kunze, H. J., \& Alexiou, S. 1996, A\&A, 307, 1023

Wrubel, Th., Ahmad, I., Bücher, S., Kunze, H. J., \& Glenzer, S. 1998, Phys. Rev. E, 57, 5972 